\title{
PRODUÇÃO DO CONHECIMENTO ACERCA DA ATENÇÃ̃O À SAÚDE DO HOMEM NO BRASIL
}

\author{
PRODUCTION OF KNOWLEDGE ABOUT HEALTH CARE OF MAN IN BRAZIL
}

\section{Leonardo Peixoto Pereira ${ }^{1 *}$, Jefferson Paixão Cardoso ${ }^{2 *}$, Adriana Alves $\mathrm{Nery}^{3^{*}}$, Alba Benemérita Alves Vilela ${ }^{* *}$}

1Ipeixotop@gmail.com, 2jpcardoso@uesb.edu.br, ${ }^{3}$ aanery@gmail.com, ${ }^{4}$ albavilela@gmail.com

*Departamento de Saúde da Universidade Estadual do Sudoeste da Bahia - UESB

Data de entrada do artigo: 11/09/2013

Data de aceite do artigo: 19/03/2014

\section{RESUMO}

Objetivo: Descrever o panorama da produção do conhecimento no Brasil acerca da atenção à saúde do homem na Atenção Básica. Metodologia: Tratou-se de uma revisão da literatura científica brasileira publicada nas bases de dados: Literatura Latino-Americana e do Caribe em Ciências da Saúde; Scientific Electronic Library Online; Bibliografia Brasileira de Odontologia; Biblioteca de Enfermagem; Hanseníase; Coleção Nacional das Fontes de Informação do Sistema Único de Saúde; Bibliografia Brasileira de Homeopatia; Index Psi Teses; Index Psi Periódicos de Divulgação Científica; Index Psi Periódicos TécnicoCientíficos e na Bibliografia em Indicadores da Saúde, no período de 10 anos compreendidos entre os anos 2002 e 2012. Consideraçóes: No panorama da produção do conhecimento no Brasil acerca da atenção à saúde do homem na Atenção Básica destaca-se a região Sudeste como o local onde há maior frequência da realização de estudos relacionados à saúde do homem, e demonstra-se a disparidade no número de estudos produzidos nas demais regióes do país. Além disso, supóe-se que o interesse e a produção do conhecimento acerca das nuances da atenção à saúde do homem estão em ascensão.

Palavras-chave: Saúde do homem; atenção primária à saúde; estudos epidemiológicos.

\section{ABSTRACT}

Objective: To describe the scenery of production of knowledge in Brazil about the health care of man in Primary Care. Methodology: This study consisted of a review of Brazilian scientific literature published in the databases: Literatura Latino-Americana e do Caribe em Ciências da Saúde (LILACS); Scientific Electronic Library Online; Bibliografia Brasileira de Odontologia; Biblioteca de Enfermagem; Hansen's disease; Coleção Nacional das Fontes de Informação do Sistema Único de Saúde; Bibliografia Brasileira de Homeopatia; Index Psi Thesis; Index Psi Scientic Journals; Index Psi Technoscientific Journals and in Bibliografia em Indicadores da Saúde, during the 10 year period from 2002 to 2012. Considerations: In the scenery of production of knowledge in Brazil on the health care of man, health in primary care stands out the Southeast as the region in which there is a greater frequency of studies related to men's health, and depicts the disparity in number of studies produced in other regions of the country. Furthermore, it is assumed that the interest and production of knowledge about the nuances of men's health care are increasing.

Keywords: Men's health; primary health care; epidemiologic studies. 


\section{Introdução}

A partir das décadas finais do século $\mathrm{XX}$, diversos países começaram a discutir os sistemas de saúde em vigência, a fim de encontrar meios de viabilizar a mudança na oferta dos serviços de saúde e de aumentar a eficiência, a eficácia e reduzir os custos da assistência de forma humanizada ${ }^{1}$.

A referida mudança o Brasil sustenta-se na Política Nacional da Atenção Básica (PNAB), que é considerada um conjunto de açôes que fomentam a promoção, a proteção à saúde, a prevenção de agravos, do diagnóstico à reabilitação e a manutenção da saúde em caráter individual e coletivo. Esta deve ser desenvolvida com o mais alto grau de descentralizaçáo e capilaridade, próxima da vida das pessoas, de modo a caracterizar-se como o centro de comunicação da Rede de Atenção à Saúde, além de considerar a singularidade, o meio sociocultural do usuário, e tem como estratégia prioritária a Estratégia de Saúde da Família $(E S F)^{2}$.

Alinhada à PNAB está a Política Nacional de Atenção Integral à Saúde do Homem (PNAISH), lançada no ano de 2009, que junto à primeira soma forças em busca de uma atenção integral à saúde, mediada por estratégias de humanizaçáo, fortalecendo assim as açóes e os serviços em redes de cuidados da saúde ${ }^{3}$.

No que diz respeito ao foco de ação da PNAISH, a pirâmide etária brasileira revela que na faixa etária de 0-19 anos há um predomínio da população masculina; entretanto, a partir dos 25 anos, a população feminina supera a masculina em todas as faixas etárias ${ }^{4}$. Entendese, deste modo, que o envelhecimento populacional tem apresentado como característica: a feminilização - mesmo nascendo mais homens no Brasil, estes estão morrendo em maior número do que as mulheres, e de forma cada vez mais precoce 5 .

Além da mortalidade, os homens são acometidos por condiçóes patológicas severas e crônicas em número maior do que as mulheres. Contudo, os homens procuram menos os serviços de atenção à saúde do que as mulheres $^{6}$.

Gomes, Nascimento e Araújo ${ }^{6}$ relacionam esta menor procura ao fato da criaçáo social do homem, visto que o cuidado não é tratado como uma prática masculina, de modo que amarras culturais e o imaginário "do que é ser homem" aprisionam o indivíduo. Afirmam ainda, que isto pode prejudicar a prevenção em saúde, pois como em uma relação de inversa proporçấo, o ser socialmente visto como forte, viril e invulnerável, ao procurar os serviços de saúde, poderá ser associado à fraqueza e à feminilidade.

Frente à supracitada menor procura, percebeu-se que os homens têm medo de adoecer, de depender de terceiros e não estão dispostos a se ausentar no trabalho.
Assim, buscam os serviços de saúde na presença de alguma doença instalada ou dor que prejudique o desempenho no trabalho, com o intuito de obter medicamentos ou analgesia, de modo que ignoram as consultas de caráter preventivo e valorizam as açóes curativas ${ }^{7,8}$.

Há muito tempo os homens usam os serviços de saúde por meio da atenção especializada, o que evidencia a necessidade de fortalecer e qualificar a atenção básica para a efetiva promoção da saúde e prevenção de agravos. Até o ano de 2009, não existiam açôes de saúde direcionadas à saúde do homem, e as demandas masculinas eram diluídas em outros programas de atenção, tais como: hipertensão, diabetes, saúde mental, saúde do trabalhador, entre outros ${ }^{9-11}$.

Neste ínterim, o estudo justificou-se pela oportunidade de reunir, sistematizar, analisar e destacar os desenhos metodológicos e eixos temáticos dos estudos, realizados em distintas realidades brasileiras, acerca da atenção à saúde do homem na Atenção Básica.

Logo, entende-se o estudo em questão como uma ferramenta capaz de organizar a produção do conhecimento no que diz respeito à atenção à saúde do homem na Atenção Básica, com o intuito de produzir material científico; incentivar a atuação profissional e incitar novos estudos, com desenhos metodológicos concisos, na busca do preenchimento das lacunas do conhecimento da referida temática.

Diante do exposto, o estudo teve por objetivo descrever o panorama da produçáo do conhecimento no Brasil acerca da atenção à saúde do homem na Atenção Básica.

\section{Metodologia}

Tratou-se de uma revisão da literatura científica brasileira publicada nas bases de dados: Literatura LatinoAmericana e do Caribe em Ciências da Saúde (LILACS); Scientific Electronic Library Online - SciELO, que tratam das Ciências em Saúde em Geral, e nas Bases Especializadas Nacionais: Bibliografia Brasileira de Odontologia (BBO); Biblioteca de Enfermagem (BDENF); Hanseníase (Hansen); Coleção Nacional das Fontes de Informação do SUS (Coleciona-SUS); Bibliografia Brasileira de Homeopatia (Homeoindex); Index Psi Teses; Index Psi Periódicos de Divulgação Científica; Index Psi Periódicos Técnico-Científicos e na Bibliografia em Indicadores da Saúde, indexadas à Biblioteca Virtual em Saúde (BVS), no período de 10 anos compreendidos entre os anos 2002 e 2012.

A escolha das bases citadas deveu-se ao fato da LILACS ser um índice bibliográfico da literatura relativa às ciências da saúde que publica nos países da América Latina e no Caribe desde 1982, e já ter ultrapassado o 
número de meio milhão de publicaçóes na modalidade artigo. Já a SciELO disponibiliza, eletronicamente, de modo "Open Access", ou seja, gratuitamente, os textos completos dos artigos de mais de 290 periódicos científicos do Brasil, Chile, Cuba, Espanha, Venezuela e outros países da América Latina ${ }^{12}$.

Em relação à inclusão das Bases Especializadas Nacionais, tratou-se de uma estratégia de ampliar o espectro da busca e incluir, na construção do panorama da produção científica brasileira acerca da atenção à saúde do homem na Atenção Básica, os títulos não indexados às bases LILACS e SciELO, tais como: teses de doutorado, dissertações de mestrado, manuais, livros, capítulos de livros, entre outros.

A busca dos artigos seguiu os seguintes critérios: na base de dados SciELO utilizou-se o descritor "Saúde do Homem", obtido no vocabulário estruturado Descritores em Ciências da Saúde (DeCS), e operacionalizado pelo operador booleano AND entre palavras e filtrado pelo campo "Palavras do Título" - o que resultou em 13 referências; para ampliar a pesquisa buscou-se o referido descritor filtrado agora pelo campo "Assunto" e obteve-se um resultado de 30 referências. Logo, na base SciELO foram selecionadas 43 publicaçóes.

$\mathrm{Na}$ LILACS seguiu-se o mesmo procedimento de busca e na primeira etapa foram encontradas $25 \mathrm{pu}-$ blicaçóes, e na segunda, 79. Assim, na base LILACS foram selecionadas 104 referências. Repetida a referida estratégia de busca nas Bases Especializadas Nacionais, obteve-se o seguinte resultado: Coleciona SUS - 03; Index Psi Teses: 16; Index Psi Periódicos de Divulgação Científica: 02; Bibliografia em Indicadores da Saúde: 02 , de modo a conferir um total de 23 itens.

Os artigos que não foram disponibilizados na íntegra foram posteriormente obtidos por completo por meio do Periódicos CAPES acessado pelo convênio com a Universidade Estadual do Sudoeste da Bahia (UESB).

Os 170 títulos foram selecionados, inseridos e organizados no software Mendeley Desktop 1.6. A partir de entáo, procedeu-se a varredura de duplicatas e a posterior leitura dos resumos, com o intuito de selecionar as referências com base nos seguintes critérios de inclusão: estudos e demais itens produzidos no Brasil, no período de 10 anos compreendidos entre 2002 e 2012. Além disso, os critérios deveriam versar direta ou indiretamente sobre a atenção à Saúde do Homem na Atenção Básica.

Após a aplicação do critério de inclusão foram selecionados 80 títulos para compor o panorama em questão. Convém ressaltar que as referências que cumpriam os critérios de inclusão e que foram publicadas em periódicos internacionais, na língua inglesa, também foram buscadas na base PUBMED, por meio do descritor "Men's Health". Entretanto, estes foram também detectados em português, e foram excluídos da contagem.

\section{Resultados e Discussão}

Para dinamizar o processo de apresentação dos resultados e discussão decidiu-se dividi-los em dois eixos de descrição: Eixo 01 - distribuição das referências por características gerais; e Eixo 02 - distribuição das referências por eixos temáticos.

Para a construçáo do panorama foram selecionadas 80 publicaçóes de referências elaboradas no Brasil. No Eixo 01 apresenta-se a distribuição das referências por características gerais, com base na Frequência Absoluta $\left(f_{\mathrm{i}}\right)$ e na Frequência Relativa $\left(f_{\mathrm{ri}}\right)$, conforme o disposto na Tabela 01 .

As seguintes características foram destacadas: o ano da publicação, compreendidos entre 2002 e 2012; a região da realização do estudo, compreendidas as macrorregiôes geográfico-políticas do Brasil (Centro-Oeste, Nordeste, Norte, Sudeste e Sul) e os aspectos básicos da metodologia empregada na realizaçáo dos estudos, e a modalidade das publicaçóes divididas em: estudos originais, artigos de opiniāo, estudos de revisáo da literatura, tese de doutorado, dissertaçáo de mestrado, editorial, manual, capítulo de livro e livro.

Neste ínterim, observa-se que a maior frequência de publicaçôes ocorreu no ano de 2011 (26,25\%) seguido dos anos 2005 (18,75\%), 2010 (17,50\%), 2009 (13,75\%), 2006 (6,25\%), 2008 (5,0\%), $2012(3,75 \%)$, 2007 (3,75\%), 2003 (2,5\%) e 2002 (2,5\%) e, por fim, o ano 2004, no qual não houve publicações selecionadas.

Quanto à distribuição por ano das publicaçôes, não há linearidade crescente ou decrescente da frequência da produção. Entretanto, cabe aqui discutir a maior concentração das publicaçóes na segunda metade da década em estudo. Acredita-se que tal concentração fundamenta-se no advento do interesse pela temática "atençâo à saúde do homem", observado com o aumento da publicação no ano de 2005, principalmente com artigos de opiniáo, expressando a necessidade da atenção integral em saúde ao homem ${ }^{13-27}$. Além disso, cita-se o lançamento da Política Nacional de Atenção Integral à Saúde do Homem (PNAISH) como o marco da influência para o aumento dos estudos da saúde do homem ${ }^{3}$.

A regiáo que apresentou a maior frequência de publicaçóes foi a Sudeste $(68,75 \%)$, seguida da região Nordeste (12,50\%), Sul (7,5\%) e Centro-Oeste (6,5\%). A regiāo Norte não apresentou publicações no escopo da pesquisa. Estudos multirregionais envolvendo a regiáo Sudeste e Nordeste representaram 3,37\%, e os que abrangeram as regiōes Sudeste, Nordeste e Sul totalizaram $1,25 \%$ dos estudos. 
Tabela 1: Distribuição por características gerais das referências acerca da atenção à saúde do homem na atenção básica, 2013

\begin{tabular}{|c|c|c|c|c|c|c|c|c|c|c|c|c|}
\hline \multicolumn{13}{|c|}{ Ano da publicaçáo } \\
\hline $\mathbf{N}$ & & 2002 & 2003 & 2004 & 2005 & 2006 & 2007 & 2008 & 2009 & 2010 & 2011 & 2012 \\
\hline \multirow[t]{2}{*}{80} & $f_{\mathrm{i}}$ & 2 & 2 & 00 & 15 & 05 & 03 & 04 & 11 & 14 & 21 & 03 \\
\hline & $f_{\mathrm{ri}}$ & 2,5 & 2,5 & 00 & 18,75 & 6,25 & 3,75 & 5,0 & 13,75 & 17,50 & 26,25 & 3,75 \\
\hline \multicolumn{13}{|c|}{ Regiáo de realizaçáo do estudo } \\
\hline $\mathbf{N}$ & & $\begin{array}{c}\text { Centro- } \\
\text { Oeste }\end{array}$ & \multicolumn{2}{|c|}{ Nordeste } & Norte & Sudeste & Sul & \multicolumn{2}{|c|}{ Multirregional I* } & \multicolumn{2}{|c|}{$\begin{array}{c}\text { Multirregional } \\
\text { II }^{*}\end{array}$} & $\begin{array}{c}\text { Multirregional } \\
\text { II }^{*}\end{array}$ \\
\hline \multirow[t]{2}{*}{80} & $f_{\mathrm{i}}$ & 05 & \multicolumn{2}{|c|}{10} & 00 & 55 & 06 & \multicolumn{2}{|c|}{03} & \multicolumn{2}{|c|}{01} & 01 \\
\hline & $f_{\mathrm{ri}}$ & 6,25 & \multicolumn{2}{|c|}{12,50} & 00 & 68,75 & 7,50 & \multicolumn{2}{|c|}{3,75} & \multicolumn{2}{|c|}{1,25} & 1,25 \\
\hline \multicolumn{13}{|c|}{ Aspectos básicos da metodologia } \\
\hline $\mathbf{N}$ & & E. Ana $^{a}$ & \multicolumn{2}{|c|}{ E. Des ${ }^{b}$} & $\mathrm{Ava}^{\mathrm{d}}$ & R. Soc ${ }^{\mathrm{e}}$ & R. $\operatorname{Exp}^{\mathrm{f}}$ & Deb $^{\mathrm{g}}$ & Res $^{\text {h }}$ & $\mathbf{R L}^{\mathrm{i}}$ & C. $\mathrm{C}^{\mathrm{j}}$ & Qnt-Qul. ${ }^{1}$ \\
\hline \multirow[t]{2}{*}{64} & $f_{\mathrm{i}}$ & 6 & \multirow{2}{*}{\multicolumn{2}{|c|}{$\begin{array}{c}7 \\
10,93\end{array}$}} & 1 & 4 & 2 & 4 & 2 & 9 & 1 & 1 \\
\hline & $f_{\mathrm{ri}}$ & 9,37 & & & 1,56 & 6,25 & 3,12 & 6,25 & 3,12 & 14,06 & 1,56 & 1,56 \\
\hline \multicolumn{13}{|c|}{ Modalidade da Publicaçáo } \\
\hline $\mathbf{N}$ & \multicolumn{5}{|c|}{ Original* } & Revisão* & Tese* & Disser.* & Edit* & Livro & Cap* & Manual \\
\hline \multirow[t]{2}{*}{80} & $f_{\mathrm{i}}$ & \multicolumn{3}{|c|}{41} & & 9 & 3 & 9 & 3 & 2 & 1 & 2 \\
\hline & $f_{\mathrm{ri}}$ & \multicolumn{3}{|c|}{51,25} & & 11,25 & 3,75 & 11,25 & 3,75 & 2,5 & 1,25 & 2,5 \\
\hline
\end{tabular}

n: Número de Referências; $f_{\mathrm{i}}$ : Frequência Absoluta; $f_{\text {ri }}$ : Frequência Relativa (\%).

Multirregional I*: Nordeste e Sudeste.

Multirregional II*: Nordeste, Sudeste e Sul.

a: Abordagem Quantitativa - Estatística analítica. ${ }^{\text {b: }}$ Abordagem Quantitativa - Estatística Descritiva. c: Abordagem Qualitativa - Análise de Conteúdo. d: Avaliação de Serviço - Técnicas de Planejamento Participativo e Avaliação Rápida. e: Representaçóes Sociais. f: Relato de Experiência. ${ }^{\text {g: }}$ Debate. ${ }^{\text {h: }}$ Resenha ${ }^{\text {i }}$ : Revisão da Literatura. J: Caso - Controle. ${ }^{\text {l: }}$ Abordagem Quantitativa e Qualitativa: Estatística Descritiva e Análise de Conteúdo.

Original*: Estudos Originais. Opinião*: Artigos de opinião Revisão*: Estudos de revisão da literatura. Tese*: Tese de Doutorado. Disser.*: Dissertação de Mestrado. Edit*: Editorial. Cap*: Capítulo de Livro.

Excetuando os diversos fatores para tal discrepância nas frequências entre as regiōes, decidiu-se enveredar a discussão pelos princípios do Sistema Único de Saúde (SUS) e citar o princípio da Universalidade (acesso igualitário aos serviços e açōes de saúde $)^{28}$, visto que a PNAISH alinha-se aos princípios do SUS ${ }^{3}$. Assim, acredita-se que o citado princípio pode estar comprometido diante da concentraçáo dos estudos, e da possibilidade do advento de serviços de atençáo ao homem, em determinadas regióes do país.

De forma mais pontual, supóe-se que a centralizaçáo das pesquisas e do desenvolvimento de serviços, no tocante à atenção à saúde do homem, principalmente em grandes centros, pode concorrer para a ocorrência de algumas doenças em outras regióes, por exemplo, o câncer de pênis, tumor raro, mais frequente nas regiōes Norte e Nordeste ${ }^{29}$.

A descrição por meio de frequências acerca das características metodológicas teve como base de cálculo apenas 64 referências, pois foram excluídas as publicações as quais não se aplicam tal descrição, tais como: livros, capítulos de livros, comentários, editoriais, entre outros.

Dessa forma, destacou-se a abordagem qualitativa com análise de conteúdo $(42,18 \%)$ e seguiu-se em ordem decrescente de frequência: a revisão de literatura (14,06\%); a abordagem quantitativa com estatística descritiva $(10,93 \%)$; a abordagem quantitativa com estatística analítica (9,37\%); representaçóes sociais $(6,25 \%)$; debate $(6,25 \%)$; relato de experiência $(3,12 \%)$; resenha $(3,12 \%)$; avaliação $(1,56 \%)$; caso-controle $(1,56 \%)$; abordagem quantitativa e qualitativa com estatística descritiva e análise de conteúdo (1,56\%).

Todas as pesquisas e demais referências descritas até o momento foram divididas nas seguintes modalidades de publicação: estudos originais (51,25\%); artigos de opinião (12,50\%); estudos de revisão da literatura (11,25\%); dissertação de mestrado (11,25\%); tese de doutorado $(3,75 \%)$; editorial $(3,75 \%)$; livro $(2,5 \%)$; manual (2,5\%); capítulo de livro (1,25\%).

A descrição dos aspectos básicos da metodologia e das modalidades da publicação permitiu a reflexão sobre uma visível mudança da produção do conhecimento a partir da segunda etapa da década em análise, não somente pelo aumento da quantidade, mas, sim, também pela alteração do tipo de publicação em ascensão nos anos seguintes. 
Acredita-se que o já citado aumento das opinióes em 2005 influenciou a ampliação do número de estudos nos anos seguintes acerca da atenção integral à saúde do homem, fundamentado na ocorrência da maior frequência de estudos originais com interesse nas questóes da saúde do homem na Atenção Básica, tais como: gênero, sexualidade, acessibilidade, serviços de saúde em atenção à saúde do homem, masculinidade, reprodução, epidemiologia, políticas em saúde, entre outros.

Uma hipótese que se levanta é que o lançamento da PNAISH, devido à essência preventiva, além de elucidativa quanto ao diagnóstico da situação da saúde do homem no Brasil ${ }^{2}$, fomentou os estudos com enfoque na Atenção Básica, na prevenção, nos serviços e na atenção à saúde do homem em geral.

Cabe aqui ressaltar o entendimento que se faz acerca da presença de teses de doutorado, dissertaçóes de mestrado, livros e capítulos de livros, bem como o uso de metodologias diversas, tais como: os estudos de caso-controle, as avaliaçóes, as análises de conteúdo, as análises estatísticas, os relatos de experiências, as representaçóes sociais, os debates, as resenhas e as revisóes da literatura, mesmo com a significativa discrepância de frequências entre estas, como resultado de um processo de construçáa da consciência coletiva das questóes de saúde do homem como uma questáo indubitável de interesse para a saúde pública, destacada de forma crescente na primeira década do século $\mathrm{XXI}^{3,6,19,30-36}$.

Neste contexto, para complementar a descrição do panorama da produçáo do conhecimento no Brasil acerca da atenção à saúde do homem na Atenção Básica descreve-se a distribuiçáo da publicação por eixos temáticos, no Eixo 02 de discussão, conforme a Tabela 02 .

Tabela 2: Distribuição das referências por eixos temáticos acerca da atenção à saúde do homem na atenção básica, 2013

\begin{tabular}{llcc}
\hline $\mathbf{n}$ & Eixos temáticos & $\boldsymbol{f}_{\mathbf{i}}$ & $\boldsymbol{f}_{\mathbf{r i}}(\%)$ \\
\hline \multirow{2}{*}{80 Relação Homem versus Serviços de } & 36 & 45 \\
& saúde & 08 & 10 \\
Gênero & 08 & 10 \\
Sexualidade & 09 & 11,25 \\
Câncer & 04 & 5 \\
DSTs* e Disfunções Sexuais & 04 & 5 \\
Mortalidade Masculina & 03 & 3,75 \\
Violência & 02 & 2,5 \\
Envelhecimento do Homem & 02 & 2,5 \\
Reprodução & 03 & 3,75 \\
Saúde Cardiovascular & 01 & 1,25 \\
Doenças Renais & & \\
\hline
\end{tabular}

n: Número de Referências; $f$ i: Frequência Absoluta;

$f$ ri: Frequência Relativa (\%).

* Doenças Sexualmente Transmissíveis.
Para tanto, após a leitura das referências, foram elaborados os seguintes eixos temáticos de agrupamento das publicações: relação homem versus serviços de saúde $3,6,8,13,14,17,19-21,24,25,32,33,35-57$; gênero ${ }^{16,26,27,43,52,58-60}$; sexualidade $^{34,61-66}$; doenças sexualmente transmissíveis (DST) e disfunções sexuais ${ }^{15,76-78}$; mortalidade masculina ${ }^{22,79-81}$, envelhecimento do homem ${ }^{83,84}$; reprodução ${ }^{45,85 \text { pa }}$; saúde cardiovascular e doenças renais ${ }^{41,86,87}$.

Entre os temas destacados, observa-se maior frequência de publicaçóes que versam acerca da relação masculina com os serviços de atenção a saúde (45\%). Neste aspecto acredita-se que tal resultado ocorre em decorrência da divulgação de dados epidemiológicos, bem como de discussôes que revelam claramente um consenso que o homem, no decorrer da vida, procura menos os serviços de saúde do que a mulher ${ }^{3,6,31}$.

Logo, entende-se que a elevada frequência destas publicaçôes seja uma busca do entendimento do fenômeno em questão, e os pontos de discussão que emergem neste contexto são: a Política Nacional de Atenção Integral à Saúde do Homem; a dinâmica de atenção à saúde do homem na Atençâo Básica; acessibilidade; a emergência das questóes de saúde do homem nas discussóes de saúde pública; educação em saúde 3̧ $18-20,30,32,36,40,46-48,54^{\text {. }}$ Outros eixos temáticos que têm íntima relação com o eixo mais frequente, o da relação entre o homem e os serviços de saúde, por exemplo, são os que debatem as questóes de gênero (10\%), a sexualidade (10\%) e a mortalidade masculina (5\%), visto que alguns estu$\operatorname{dos}^{6,10,27,31,42,43,52,53,61,63,66}$ destacam a estreita relação entre questóes de gênero e sexualidade com a procura do homem pelos serviços de saúde, principalmente a Atenção Básica - revelando que amarras culturais afastam os indivíduos das açôes e serviços de saúde. Já outras referências ${ }^{3,16,18,22,25,30,79-81}$ demonstram que a mortalidade masculina é maior do que a feminina, discutindo e investigando as razóes deste fato, além de proporem paralelos entre a mortalidade masculina e os fatores sociais e os serviços de atenção à saúde do homem.

Convém ressaltar o destaque dado às nuances da violência no âmbito social masculino como uma questão que se relaciona aos processos de acessibilidade do homem às ações de saúde; com a estruturaçáo da política de atenção à saúde do homem e com o entendimento que este indivíduo faz do processo saúde/doença. Além de evidenciar, inquestionavelmente, a própria violência em si como um problema de saúde pública ${ }^{13,17,23,81,82}$.

No tocante às doenças que acometem o homem, tem destaque no panorama em questão: o câncer $(11,25 \%)$, principalmente o de próstata, as DSTs e disfunçóes sexuais $(5 \%)$, as doenças cardiovasculares $(3,75)$ e as doenças renais $(1,25 \%)$. Cabe aqui esclarecer que as frequências dos eixos referentes às doenças não dizem respeito à incidência destas na população masculina, 
mas referem-se às publicaçóes selecionadas que se relacionam com a atenção à saúde do homem na questão da Atenção Básica, de forma direta ou indireta. Nesta vertente as questóes de maior destaque e discussão tratam da prevençáo, dos fatores preveníveis, do sentimento do homem quando se descobre portador de alguma doença e do tratamento das doenças ${ }^{15,62,64,65,67-71,73,74,76,77,84}$.

Por fim, o panorama apresenta dois temas em emergência na produçáo do conhecimento acerca da saúde do homem: os aspectos do envelhecimento do homem ${ }^{83,84}$ e a inserção deste indivíduo no contexto da reprodução humana ${ }^{45,85}$.

Assim, parte-se do pressuposto de que a política de atenção à saúde do homem pretende, entre outros anseios, aumentar a expectativa de vida do homem brasileiro, bem como aliar tal aumento à qualidade de vida e manutenção da capacidade funcional ${ }^{3}$. Sendo assim, entende-se a necessidade de produzir conhecimento acerca do envelhecimento masculino como uma estratégia aliada à promoção de saúde do homem.

Em relação à reprodução, os estudos versam sobre a responsabilizaçáo masculina no processo reprodutivo, principalmente em situaçóes conflitantes, tais como: abortos, doaçóes de filhos e adoção, além de trazer à tona as questóes da saúde reprodutiva do homem, no intuito de esclarecê-las e fomentar açóes de prevenção em saúde reprodutiva, de forma que o homem seja reconhecido como um ator social integrante de todo o processo reprodutivo ${ }^{45,85}$.

Considera-se como limitação do estudo e da discussão, o fato de que os artigos aceitos para publicação, mas ainda não publicados, não podem ser detectados pelas ferramentas metodológicas disponíveis para a realização desta pesquisa.

\section{Considerações Finais}

No panorama da produção do conhecimento no Brasil acerca da atençáo à saúde do homem na Atenção Básica, destaca-se a região Sudeste como o local onde há a maior frequência da realizaçáo de estudos relacionados à saúde do homem e demonstra-se a disparidade no número de estudos produzidos nas demais regióes do país, o que suscita a preocupação com a regionalização da produçáo do conhecimento e com a implementação de açóes e serviços de Atenção Básica à saúde do homem em regiōes distantes do Sudeste, principalmente na região Norte.

Fundamentado no aumento de estudos originais e associado à variedade de metodologias utilizadas nas pesquisas a partir da segunda metade da década em questão, em detrimento das publicaçôes de opiniāo, considera-se que o interesse e a produção do conhecimento acerca das nuances da atenção à saúde do homem estão em ascensão.

Neste ínterim, percebeu-se que atualmente o foco de estudo dos pesquisadores em saúde do homem está ancorado no entendimento da relação homem versus serviços de saúde, no estudo do câncer com enfoque na saúde do homem, principalmente o de próstata, assim como nas questóes de gênero e sexualidade, seguido pelo interesse nas questóes que envolvem as DSTs e disfunçóes sexuais, mortalidade masculina, violência, saúde cardiovascular e doenças renais. Além disso, foi possível ressaltar a emergência de temas como o envelhecimento masculino e a participação masculina nos aspectos da reprodução.

\section{Referências}

1. Copque HLF, Trad LAB. Programa Saúde da Família: a experiência de implantação em dois Municípios da Bahia. Epidemiol. Serv. Saúde. 2005; 14(4):223-33.

2. Brasil. Ministério da Saúde. Secretaria de Atenção à Saúde. Departamento de Atenção Básica. Portaria no 2.488, de 21 de Outubro de 2011. Política Nacional de Atenção Básica. Diário Oficial da União. Brasília, 24 out. 2011; Seção 1. p. 48.

3. Brasil. Ministério da Saúde. Secretaria de Atenção à Saúde. Departamento de Atenção Básica. Portaria 1.944 de 27 de agosto de 2009. Política Nacional de Atenção Integral à Saúde do Homem: princípios e diretrizes. Diário Oficial da União. Brasília, 28 ago. 2009; Seção 1. p.61.

4. Instituto Brasileiro de Geografia e Estatística. Censo 2010. IBGE [internet]. 2010. Disponível em: <http://www.censo2010.ibge.gov.br/apps/mapa/>

5. Lourenço RA, Lins RG. Saúde do homem: aspectos demográficos e epidemiológicos do envelhecimento masculino. Revista do Hospital Universitário Pedro Ernesto. 2010; 9(1):12-9.

6. Gomes R, Nascimento EF, Araújo FC. Por que os homens buscam menos os serviços de saúde do que as mulheres? As explicaçóes de homens com baixa escolaridade e homens com ensino superior. Cad. Saúde Pública. 2007; 23(3):565-74.

7. Albano B, Basílio M, Neves J. Desafios para a Inclusão dos homens nos serviços de Atenção Primária a Saúde. Revista Enfermagem Integrada. 2010; 3(2):554-536.

8. Schraiber LB, Figueiredo WS, Gomes R, Couto MT, Pinheiro TF, Machin R, et al. Necessidades de saúde e masculinidades: atenção primária no cuidado aos homens. Cad. Saúde Pública. 2010; 26(5):961-70.

9. Julião G, Weigelt L. Atenção à Saúde do Homem em Unidades de Estratégia de Saúde da Família. Rev Enferm UFSM. 2011; 1(2):144-52.

10. Couto MT, Pinheiro TF, Valença O, Machin R, Silva GSN da, Gomes R, et al. O homem na atenção primária à saúde: 
discutindo (in)visibilidade a partir da perspectiva de gênero. Interface - Comunicação, Saúde, Educação. 2010; 14(33):257-70.

11. Dominguez B. Saúde do Homem: hora de quebrar paradigmas. Radis Comunicação em Sáude. 2008; 74(1):8-9.

12. Biblioteca Virtual em Saúde. [internet]. 2012; 1-2. Disponível em: <http://regional.bvsalud.org/php/index. php>

13. Anon. Os autores respondem. Ciênc. saúde colet [internet]. 2005; 10(1); 32-4. Disponível em: <http://www.scielo.br/scielo. php?script=sci_arttext\&pid=S1413-81232005000100009>

14. Aquino EML. Saúde do homem: uma nova etapa da medicalização da sexualidade? Ciênc. saúde colet. 2005; 10(1):19-22.

15. Araújo MAL, Leitão GCM. Acesso à consulta a portadores de doenças sexualmente transmissíveis: experiências de homens em uma unidade de saúde de Fortaleza, Ceará, Brasil. Cad. Saúde Pública. 2005; 21(2):396-403.

16. Braz M. A construção da subjetividade masculina e seu impacto sobre a saúde do homem: reflexão bioética sobre justiça distributiva. Ciênc. saúde colet. 2005; 10(1):97-104.

17. Couto MT, Schraiber LB. Homens, saúde e violência: novas questôes de gênero no campo da saúde coletiva. In: Minayo MCS, Coimbra Júnior CEA (Org.). Críticas e atuantes: ciências sociais e humanas em saúde na América Latina. Rio de Janeiro: Fiocruz; 2005. p. 687-706.

18. Figueiredo W. Assistência à saúde dos homens: um desafio para os serviços de atenção primária. Ciênc. saúde colet. 2005; 10(1):105-9.

19. Gomes R, Schraiber LB, Couto MT. O homem como foco da Saúde Pública. Ciênc. saúde colet. 2005; 10(1):4-4.

20. Knauth DR, Machado PS. Comentários diagonais sobre a emergência dos homens na pauta da Saúde Coletiva. Ciênc. saúde colet. 2005; 10(1):28-9.

21. Knauth DR, Machado PS. Comentários ao artigo "Homens e saúde na pauta da Saúde Coletiva." Ciênc. saúde colet. 2005; 10(1):18-9.

22. Laurenti R, Jorge MHPM, Gotlieb SLD. Perfil epidemiológico da morbimortalidade masculina. Ciênc. saúde colet. 2005; 10(1):35-46.

23. Minayo MCS. Laços perigosos entre machismo e violência. Ciênc. saúde colet. 2005; 10(1):23-6.

24. Nascimento P. Homens e saúde: diversos sentidos em campo. Ciênc. saúde colet. 2005; 10(1):26-8.

25. Schraiber LB, Gomes R, Couto MT. Homens e saúde na pauta da Saúde Coletiva. Ciênc. saúde colet. 2005; 10(1):7-1.

26. Silva MMA. O masculino em conferências e programas das Naçôes Unidas: para uma crítica do discurso de gênero. São Paulo; 2005. Doutorado [Tese].Universidade de São Paulo.

27. Villela W. Gênero, saúde dos homens e masculinidades. Ciênc. saúde colet. 2005; (1):29-32.
28. Paim JS, Silva LMV. Universalidade, integralidade, equidade e SUS. BIS, Bol. Inst. Saúde. 2010; 12(2):109-14.

29. Nardi A, Glina S, Favorito L. Primeiro estudo epidemiológico sobre câncer de pênis no Brasil. International Braz J Urol. 2007; 33(1):1-7.

30. Fontes WDD, Barboza TM, Leite MC, Fonseca RLS, Santos LCF, Nery TCL. Atenção à saúde do homem: interlocução entre ensino e serviço. Acta Paul. Enferm. 2011; 24(3):430-3.

31. Gomes R, Moreira MCN, Nascimento EF, Rebello LEFS, Couto MT, Schraiber LB. Os homens não vêm! Ausência e/ou invisibilidade masculina na atenção primária. Ciênc. saúde colet. 2011; 16(Suppl. I):983-92.

32. Gomes R, Nascimento EF. A produção do conhecimento da saúde pública sobre a relação homem-saúde: uma revisão bibliográfica. Cad. Saúde Pública. 2006; 22(5):901-11.

33. Magnani C, Dias JCP, Gontijo ED. Como as açôes de saúde pensam o homem e como o homem as repensa: uma análise antropológica do controle da doença de Chagas. Cad. Saúde Pública. 2009; 25(9):1947-56.

34. Pinheiro TF, Couto MT, Silva GSN. Questóes de sexualidade masculina na atenção primária à saúde: gênero e medicalização. Interface comun. saúde educ. 2011; 15(38):845-58.

35. Rebello L. Saúde do homem em debate. Cad. Saúde Pública. 2012; 28(6):1213-1213.

36. Separavich MA. A saúde do homem em foco. Interface comun. saúde educ. 2011; 15(38):957-62.

37. Brasil. Ministério da Saúde. Secretaria de Atenção à Saúde. Departamento de Atenção Básica. Política Nacional de Atenção Integral à Saúde do Homem: plano de ação nacional. Brasília, 30 jul. 2009. Disponível em: <http://portal. saude.gov.br/portal/arquivos/pdf/apresentacao_saude_homem_0709.pdf>

38. Brito RS, Santos DLA, Maciel PSO. Olhar masculino acerca do atendimento na estratégia saúde da família. Rev. Min. Enferm. 2010; 11(4):135-42.

39. Bursztyn I. Estratégias de mudança na atenção básica: avaliação da implantação piloto do Projeto Homens Jovens e Saúde no Rio de Janeiro, Brasil. Cad. Saúde Pública. 2008; 24(10):2227-38.

40. Carrara S, Russo JA, Faro L. A política de atenção à saúde do homem no Brasil: os paradoxos da medicalização do corpo masculino. Physis. 2009; 19(3):659-78.

41. Chagas ACP, Zilli EC, Ferreira JFM, Moretti MA, Ramos RF. Saúde cardiovascular do homem brasileiro: visão da Sociedade Brasileira de Cardiologia. Arq. Bras. Med. Vet. Zootec. 2009; 93(6):584-7.

42. Figueiredo WS. Masculinidades e cuidado: diversidade e necessidades de saúde dos homens na atenção primária. São Paulo; 2008. Doutorado [Tese]. Faculdade de Medicina da Universidade de São Paulo.

43. Figueiredo WS, Schraiber LB. Concepçóes de gênero de homens usuários e profissionais de saúde de serviços de 
atenção primária e os possíveis impactos na saúde da população masculina, São Paulo, Brasil. Ciênc. saúde colet. 2011; 16(Suppl.1):935-44.

44. Freitas NNS, Biagolini REM. Conhecendo o perfil e a utilização dos serviços de saúde pelos homens. Nursing (São Paulo). 2011; 14(157):328-33.

45. Galastro EP, Fonseca RMGS. A participaçáo do homem na saúde reprodutiva: o que pensam os profissionais de saúde. Rev Esc Enferm USP. 2007; 41(3):454-9.

46. Gomes R, Rebello LEFS, Nascimento EF do, Deslandes SF, Moreira MCN. A atenção básica à saúde do homem sob a ótica do usuário: um estudo qualitativo em três serviços do Rio de Janeiro. Ciênc. saúde colet. 2011; 16(11):4513-21.

47. Gomes R, Schraiber LB, Couto MTMT, Augusto O, Valença OAA, Silva GSN da, et al. O atendimento à saúde de homens: estudo qualitativo em quatro estados Brasileiros. Physis (Rio J.). 2011; 21(1):113-27.

48. Leite DF, Ferreira IMG, Souza MS, Nunes VS, Castro PR. A influência de um programa de educação na saúde do homem. Mundo saúde. 2010; 34(1):50-6.

49. Meirelles RMR, Hohl A. Saúde masculina: tão negligenciada, principalmente pelos homens. Arq Bras Endocrinol Metab. 2009; 53(8):899-900.

50. Moreira MEL, Goldani MZ. Child is the father of man: new challenges for child health. Ciênc. saúde colet. 2010; 15(2):321-7.

51. Nascimento ARA, Trindade ZA, Nascimento IFG. Homens brasileiros jovens e representaçôes sociais de saúde e doença. Psico-USF. 2011; 16(2):203-13.

52. Nascimento ARA, Trindade ZA, Nascimento IFG, Pereira FB, Silva SATC, Cerello AC. Masculinidades e práticas de saúde na regiáo metropolitana de Belo Horizonte, MG. Saúde Soc. 2011; 20(1):182-94.

53. Pinheiro TF. A abordagem à sexualidade masculina na atenção primária à saúde: possibilidades e limites. Interface Comun. Saúde Educ. 2011; 15(36):326-326.

54. Santana EN, Lima EMM, Bulhóes JLF, Monteiro EMLM, Aquino JMA atenção à saúde do homem: ações e perspectivas dos enfermeiros. Rev. Min. Enferm. 2011; 15(3):324-32.

55. Santos FA. Dinâmica da acessibilidade masculina ao Programa de Saúde da Família. Jequié; 2010. Mestrado [Dissertação]. Universidade Estadual do Sudoeste da Bahia.

56. Gomes R. A Saúde do Homem em Foco. São Paulo: Unesp; 2010.

57. Gomes R. Saúde do Homem em debate. Rio de Janeiro: Fiocruz; 2011.

58. Burille A. Itinerários terapêuticos de homens em situação de adoecimento crônico: (des)conexôes com o cuidado e arranhaduras da masculinidade. Porto Alegre; 2012. Mestrado [Dissertação]. Universidade Federal do Rio Grande do Sul.

59. Dias A, Chiavegatto P. O sexo masculino vulnerável: razáo de masculinidade entre os óbitos fetais brasileiros. Cad. Saúde Pública. 2012; 28(4):720-8.

60. Machin R, Couto MT, Silva GSN, Schraiber LB, Gomes
R, Santos Figueiredo W, et al. Concepçóes de gênero, masculinidade e cuidados em saúde: estudo com profissionais de saúde da atenção primária. Ciênc. Saúde Colet. 2011; 16(11):4503-12.

61. Gomes R. Sexualidade masculina e saúde do homem: proposta para uma discussão. Ciênc. Saúde Colet. 2003; 8(3):825-9.

62. Laydner HK, Oliveira P, Andrade WS, Santos MT, Araújo JLR. Disfunção erétil: um marcador precoce de doenças cardiovasculares. Rev. Bras. Med. 2009; 66(10):331-334.

63. Leal AF, Knauth DR. A relaçấo sexual como uma técnica corporal: representaçóes masculinas dos relacionamentos afetivo-sexuais. Cad. Saúde Pública. 2006; 22(7):1375-84.

64. Matheus WE, Fregonesi A, Ferreira U. Disfunção erétil. Rev. Bras. Med. 2009;66(12).

65. Melnik T. Ejaculaçâo precoce ou rápida. Rev. Diagn. Tratamento. 2010; 15(2):91.

66. Rebello LEFS. Iniciação sexual e masculinidade: uma análise das narrativas de homens jovens. Rio de Janeiro; 2006. Mestrado [Dissertação].Fundação Oswaldo Cruz.

67. Amorim VMSL, Barros MBA, César CLG, Goldbaum M, Carandina L, Alves MCGP. Fatores associados à realização dos exames de rastreamento para o câncer de próstata: um estudo de base populacional. Cad. Saúde Pública. 2011; 27(2):347-56.

68. Crippa A, Dall'Oglio MF, Antunes AA, Srougi M. Hiperplasia benigna da próstata. Rev. Bras. Med. 2010; 67(1/2).

69. Evangelista R. Câncer e o imaginário masculino: a expressão da doença para o homem. São Paulo; 2002. Doutorado [Tese]. Universidade de São Paulo.

70. Leme LHS, Souza GA. Câncer de mama em homens: aspectos epidemiológicos, clínicos e terapêuticos. Rev. Ciênc. Méd. 2006; 15(5):391-8.

71. Medeiros AP, Menezes MFB, Napoleão AA. Fatores de risco e medidas de prevençấo do câncer de próstata: subsídios para a enfermagem. Rev. Bras. Enferm. 2011; 64(2):385-8.

72. Mesquita MGR. Necessidades de ajuda de homens em tratamento antineoplásico: subsídios ao gerenciamento do cuidado de enfermagem. Rio de Janeiro; 2008. Mestrado [Dissertaçáo]. Universidade Federal do Rio de Janeiro.

73. Mesquita MGR, Moreira MC, Maliski S. Em busca de conhecimento de enfermagem sobre o homem com câncer: uma experiência internacional. Esc. Anna Nery Rev. Enferm. 2009; 13(2):421-4.

74. Paiva EP, Motta MCS, Griep RH. Barriers related to screening examinations for prostate cancer. Rev. Latino-Am. Enfermagem. 2011; 19(1):73-80.

75. Paiva EPD, Motta MCSD, Griep RH. Conhecimentos, atitudes e práticas acerca da detecçấo do câncer de próstata. Acta Paul. Enferm. 2010; 23(1):88-93.

76. Marianelli R, Nadal SR. Utilidade da citologia anal no rastreamento dos homens heterossexuais portadores do HPV genital. Rev. Bras. Coloproct. 2010; 30(3):365-7. 
77. Queiroz DT, Braga VAB, Ximenes LB. Homens portadores do papilomavírus humano: reaçóes emocionais na confirmação do diagnóstico. Rev. Enferm. UERJ. 2006; 14(3):405-11.

78. Rebello LEFS, Gomes R, Souza ACB. Homens e a prevençáo da aids: análise da produção do conhecimento da área da saúde. Interface Comun. Saúde Educ. 2011; 15(36):67-78.

79. Lacerda CB. Evolução da mortalidade em homens adultos no Mato Grosso do Sul, 1996 - 2007. Rio de Janeiro; 2010. Mestrado [Dissertação]. Escola Nacional de Saúde Pública Sergio Arouca.

80. Luizaga CTM. Mortalidade masculina no tempo e no espaço. São Paulo; 2010. Mestrado [Dissertação]. Universidade de São Paulo.

81. Melo EM de, Côrtes M da CJW, Miranda PSC, Câmara ACS, Alves RA, Pereira VOM, et al. Eles morrem mais do que elas. Por quê? Rev. méd. Minas Gerais. 2008; 18(4, Suppl. 4):S12-S18.

82. Lima DC, Büchele F. Revisão crítica sobre o atendimento a homens autores de violência doméstica e familiar contra as mulheres. Physis. 2011; 21(2):721-43.
83. Barbosa CRM. Saúde, qualidade de vida e envelhecimento: a inclusão do homem idoso em programas para a terceira idade. Rio de Janeiro; 2003. Mestrado [Dissertação]. Universidade Federal do Rio de Janeiro.

84. Mestrinho BV, Gomes L, Almeida JLT, Almeida JC, Oliveira, RVL. Esclarecer o idoso sobre o exame digital retal diminui o desconforto na sua primeira realização? Rev. Col. Bras. Cir. 2011; 38(6):407-11.

85. Silva CAA da, Moraes AJP, Leal MM, Sallum AME, Bonfá E, Borges CTL, et al. Aspectos da saúde reprodutiva em homens com miopatia inflamatória idiopática: um estudo multicêntrico. Rev. Bras. Reumatol. 2009; 49(6):677-89.

86. Eyken EBBDV, Moraes CL. Prevalência de fatores de risco para doenças cardiovasculares entre homens de uma população urbana do Sudeste do Brasil. Cad. Saúde Pública. 2009; 25(1):111-23.

87. Mussi FC, Koizumi MS, Angelo M, Lima MS. Perda da espontaneidade da ação: o desconforto de homens que sofreram infarto agudo do miocárdio. Rev Esc Enferm. USP. 2002; 36(2):115-24. 